\title{
Comparative Study of Antibacterial Activity of Different Essential Oils against Different Bacteria
}

\author{
Sharmin Akter ${ }^{1}$, Anika Bushra Moumita ${ }^{2}$, Tasnia Ahmed ${ }^{3}$ \\ M.S. in Microbiology Student, Department of Microbiology, Stamford University Bangladesh, Dhaka, Bangladesh ${ }^{1}$ \\ M.S. in Microbiology Student, Department of Microbiology, Stamford University Bangladesh, Dhaka, Bangladesh ${ }^{2}$ \\ Senior Lecturer, Department of Microbiology, Stamford University Bangladesh, Dhaka, Bangladesh ${ }^{3}$
}

\begin{abstract}
The problem caused by drug resistant pathogenic bacteria has become a common phenomenon from the last few decades. People infected with many bacteria which was once easily curable by the administration of proper antibiotics, have become difficult to eradicate due to their acquisition of antibiotic resistant trait. As a result people are dying from lack of proper medication which will be able to kill such pathogens. In search of such potent therapeutics, natural resources are targeted and essential oil is such a natural resource which can actively inhibit different pathogenic bacteria. Current study aimed in to determine the potency of tea tree essential oil, sweet orange essential oil and ylangylang essential oil against antibiotic resistant bacteria. The efficacy of the essential oils was determined against Staphylococcus aureus, Pseudomonas luteola, Pseudomonas aeruginosa, Klebsiella pneumonia, Bacillus subtilis and Escherichia coli and was compared by using both micro dilution and agar well diffusion method. The highest average inhibition rate was found for tea tree essential oil (93.67\%) followed by ylang-ylang (81.33\%) and sweet orange essential oil (74.16\%) after twenty four hours. Tea tree oil was $100 \%$ effective against all bacteria used in this experiment except Bacillus subtilis (62\%). Highest percentage inhibition for ylang-ylang was against Klebsiella pneumoniae (100\%). Sweet orange essential exhibited $100 \%$ inhibition for Klebsiella pneumoniae, Pseudomonas luteola and Escherichia coli. Tea tree oil has the highest ability to inhibit all of the six selected bacteria which are already resistant to antibiotics. It has been observed that multi drug resistant isolates (Escherichia coli- resistant to $4^{\text {th }}$ generation cephalosporin) is surprisingly inhibited by all of these essential oils. The antibacterial properties of these oils can be aimed to produce new therapeutics to combat the resistant bacteria.
\end{abstract}

Keywords: Essential Oil, Pathogenic Bacteria, Antibacterial Activity, Drug Resistance, Percentage Inhibition

\section{INTRODUCTION}

Essential oils are generally secondary plant metabolites extracted from different parts of a plant like fruit, flowers, leaves, seeds, barks, stems etc. belonging to the plant families of Aristolochiaceae, Meliaceae, Asteraceae, Rutaceae, Fabaceae, Lamiaceae, Cupressaceae, Myrtaceae, Lauraceae etc.[1,2]. They can be stored in canals, cavities, secretory cells or glandular ,trichomes [3]. These oils can serve in medical treatment as antimicrobial, antiviral, anticancer, antiinflammatory, antiparasitic, insecticidal, antifungal, as analgesic products and even as food preservatives as well as active ingredients in cosmetics [4-13]. As many disease causing bacteria have become multiple drug resistant, they are hardly resistible by using conventional antibiotics and as a result the morbidity and mortality rate is increasing because of infections which were once easy to treat [14-18]. So seeking for new antimicrobial agents is continuing from natural sources which can work against a wide range of bacteria including the multi drug resistant species and essential oils have become a good candidate for such activities [19-24]. Essential oils are comprised of a mixture of low molecular weight volatile compounds like terpenes, aromatic and aliphatic phenols and aldehydes, alcohol, acyclic esters, acids, isoprenoids, lactones [25, 26]. Essential oil extracted from sunflower, cinnamon bark, lemongrass, clove, geranium, lemon, lime, orange and rosemary, camphor, sweet basil, breckland thyme, lavender, oregano, olive, mustard, eucalyptus oil, neem, anis, cumin, mint have been showed to possess antimicrobial properties in different studies against many pathogenic bacterial isolates [27].

In present study we selected three essential oils like tea tree oil, sweet orange oil and ylang ylang oil. Tea Tree (Melaleuca alternifolia) essential oil has been studied against several pathogenic antibiotic resistant bacteria and found to be effective in inhibiting them quite efficiently [28-30]. Though it is toxic for consumption or injection, it works best as topical formulations (for acne, dandruff or any other skin infection) to impart its antibacterial activity [31, 32]. Tea tree oil increases the cellular permeability of liposomal systems encouraging their lysis due to the leakage of ions as well as inhibition of respiration resulting in the desired death of the bacterium [33]. Similarly sweet orange (Citrus sinensis) also possess some antimicrobial and antioxidant properties [34-36]. Ylang-ylang (Cananga odorata) exhibits 


\section{International Advanced Research Journal in Science, Engineering and Technology}

Vol. 6, Issue 6, June 2019

antibacterial, antifungal and antiplasmodial activities which showed better inhibitory effect towards Gram-positive bacteria rather than Gram-negative bacteria [37-39]. Current study was aimed to determine the antibacterial properties of Tea Tree Oil (Melaleuca alternifolia) (TTO), Sweet Orange Oil (Citrus sinensis) (SOO) and Ylang-ylang (Cananga odorata) oil against six bacterial isolates (Bacillus subtilis, Escherichia coli, Staphylococucs aureus, Pseudomonas aeruginosa, pseudomonas luteola, Klebsiella pneumonia) and to compare the antibacterial properties of these three essential oils.

\section{MATERIALS AND METHOD}

\section{Study area and sampling}

The study included commercially available three essential oil samples- Tea Tree Oil (Melaleuca alternifolia) (TTO), Sweet Orange Oil (Citrus sinensis) (SOO) and Ylang-ylang (Cananga odorata) oil collected from super shops in Dhaka metropolis. The study was done during the time span of January, 2019 to March, 2019. Samples were collected aseptically from commercial sealed bottles and antimicrobial assay was conducted against six different bacterial isolates.

\section{Test organisms}

Six different bacterial isolates were collected from different sources to analyze the antibacterial activity of Tea Tree Oil (Melaleuca alternifolia) (TTO), Sweet Orange Oil (Citrus sinensis) (SOO) and Ylang-ylang (Cananga odorata) oil. The bacteria isolates used for this study included Pseudomonas luteola, Pseudomonas aeruginosa, Escherichia coli, Klebsiella pheumoniae (collected from hospitalizes patients), Bacillus subtilis (collected from soil environmental sample), Staphylococcus aureus (collected from the collection of clinical freeze dried laboratory isolates). All of these bacteria were identified following standard biochemical tests.

\section{Antibiotic susceptibility of the tested organisms}

To determine the susceptibility of the bacterial isolates towards some commonly prescribed antibiotics was revealed by agar disc-diffusion method called the Kirby Bauer method. About 25 antibiotics were used including Amikacin (30 $\mu$ g), Cefepime (30 $\mu \mathrm{g})$, Gentamycin $(10 \mu \mathrm{g})$, Colistin $(10 \mu \mathrm{g})$, Nitrofurantoin $(50 \mu \mathrm{g})$, Cephradine $(30 \mu \mathrm{g})$, Ceftriaxone $(30 \mu \mathrm{g})$, Rifampin $(5 \mu \mathrm{g})$, Novobiocin $(30 \mu \mathrm{g})$, Nalidixic Acid $(30 \mu \mathrm{g})$, Amoxicillin $(30 \mu \mathrm{g})$, Ampicillin $(10 \mu \mathrm{g})$, Cefepime $(30 \mu \mathrm{g})$, Cefoperazone, Tigecycline, Piperacillin/Tazobactam, Meropenem, Imipenem, Ciprofloxacin (5 $\mathrm{gg})$, Trimethoprim/Sulfamethoxazole $(25 \mu \mathrm{g})$, Entrapenem $(10 \mu \mathrm{g})$, Cefpodoxime $(30 \mu \mathrm{g})$, Neomycin $(30 \mu \mathrm{g})$, Erythromycin $(30 \mu \mathrm{g})$, Tetracycline $(30 \mu \mathrm{g})$. After standardizing with $0.5 \mathrm{McFarland}$ solution suspensions of Klebsiella pheumoniae, Escherichia coli, Staphylococcus aureus, Pseudomonas luteola, Pseudomonas aeruginosa, Bacillus subtilis were prepared. Lawn of the bacterial suspension was prepared on Mueller-Hinton agar plates using sterile cotton swab individually for each bacteria. Antibiotic discs were placed over the surface of the inoculated Mueller-Hinton agar plates aseptically and incubated for 8 hours at $37^{\circ} \mathrm{C}$. After the period of incubation, all the plates were observed for the presence of the clear zone of inhibition and measured in $\mathrm{mm}$ afterwards.

\section{Detection of inhibition percentage}

At first one tube containing $5 \mathrm{ml}$ of Brain Heart Infusion broth (BHIB) and one tube containing $4 \mathrm{ml}$ of Brain Heart Infusion broth (BHIB) were taken with $1 \mathrm{ml}$ of essential oil in the second tube. Bacterial suspension $(10 \mu \mathrm{l})$ prepared earlier was inoculated into each tube. After vortex mixing the tubes were incubated for 24 hours at $37^{\circ} \mathrm{C}$. Inhibition of bacterial growth was observed at two time intervals- after 6 hours and after 24 hours respectively. After 6 hours, dilution was prepared by taking $900 \mu \mathrm{l}$ of normal saline in 5 test tubes for BHIB without oil and 3 test tubes for BHIB with oil. $100 \mu \mathrm{l}$ inoculum from normal BHIB was taken for serial dilution in $900 \mu \mathrm{l}$ normal saline and transferred 100 $\mu 1$ to the rest of the tubes till $5^{\text {th }}$ tube and discarded $100 \mu$ l. By the same way dilution was done by taking $100 \mu 1$ inoculum from BHIB with oil and diluted till $3^{\text {rd }}$ tube. Then $100 \mu$ l sample from each diluted tubes were plated onto nutrient agar plates. The plates were then incubated for 24 hours at $37^{\circ} \mathrm{C}$ and observed for the difference of bacterial growth (CFU-Colony Forming Unit) for both incubation with oil and without oil. CFU for with oil and without oil were then counted and compared to determine the percentage inhibition. Same procedure was applied after 24 hours too. The whole process was done separately for all the six bacterial isolates as well as all three types of essential oils.

\section{Antibacterial activity of direct extracts by agar well diffusion method}

Six bacterial suspensions were prepared after inoculating the isolates into normal saline followed by incubation at $37^{\circ} \mathrm{C}$ until matched with the McFarland turbidity standard $\left(10^{8} \mathrm{CFU} / \mathrm{ml}\right)$. On the Muller Hinton agar media bacterial lawn was prepared using sterile cotton swab separately for each of six bacterial suspensions respectively. Each of the three essential oil samples were then inoculated (about $100 \mu \mathrm{l}$ ) separately in the holes made in MHA afar plates using sterile cork borer. Plates were incubated for 24 hours at $37^{\circ} \mathrm{C}$ and then the presence of clear zone around the sample solution was measured in mm. 


\title{
International Advanced Research Journal in Science, Engineering and Technology
}

\author{
Vol. 6, Issue 6, June 2019
}

\section{RESULTS}

To determine the antibiotic susceptibility towards the commonly prescribed antibiotics, Kirby-Bauer antibiotic susceptibility test was performed. 25 antibiotics from different groups were selected for antibiotic susceptibility test of the six selected bacterial isolates (Table 2). Each bacterium was biochemically identified (Table 1). For each bacterium separate antibiotics were used upon the availability of antibiotics during the study. Amikacin, Cefoperazone/Sulbactam, Imipenem, Piperacillin/Tazobactam, Meropenem antibiotics were tested for four isolates which showed to be effective against all them. Gentamicin was effective for all of six isolates under investigation. Cefpodoxime, Neomycin, Tetracycline, Erythromycin were used only for environmental and laboratory isolates Bacillus subtilis and Staphylococcus aureus which found to be capable of inhibiting growth around the disc showing clear zone. Bacillus subtilis and Staphylococcus aureus were also susceptible for Cephradine, Ampicillin and Rifampicin,. The pathogenic isolates Pseudomonas luteola (Colistin), Pseudomonas aeruginosa (Tigecycline), Escherichia coli (Cefepime, Nalidixic acid, Ceftriaxone, Ciprofloxacine, Cefuroxime), Klebsiella pneumonia (Ampicillin) showed resistance to various antibiotics. Only Escherichia coli showed higher degrees of resistance against five antibiotics including $3^{\text {rd }}$ and $4^{\text {th }}$ generation cephalosporins. Total viable bacterial count was determined by incubation the isolates of Pseudomonas aeruginosa, Pseudomonas luteola, Staphylococcus aureus, Klebsiellla pneumonia, Escherichia coli and Bacillus subtilis separately in BHIB (Brain Heart Infusion Broth) and BHIB with oils (TTO,SOO, Ylang-ylang oil) separately (Table 3-Table 8). In all cases, the growth inhibited with the presence of essential oil. Then the percentage inhibition of the essential oil was determined by using the following equation.

$$
\begin{gathered}
\mathrm{CFU}=\frac{\text { Number of colonies } \times \text { reciprocal of the dilution factor }}{\text { Volume of plated suspension }} \\
\text { Inhibition percentage }=1-\left(\frac{\mathrm{CFU} \text { in oil }}{\mathrm{CFU} \text { in broth }}\right) \times 100
\end{gathered}
$$

The inhibition percentage was found to be highest with TTO and SOO for all bacteria except Bacillus subtilis after 24 hours (Table 9). Ylang-ylang oil showed the lowest inhibition percentage for Pseudomonas luteola and Bacillus subtilis. Average percentage inhibition was the calculated and found out (Table 10) to be quite effective for all the essential oils after 24 hours. This result is easily understandable by the graph of Figure 1. Here it has been seen that TTO showed the best result in inhibiting bacteria on average than the other two oils. SOO showed the minimum average inhibitory activity leaving Ylang- ylang oil in between of TTO and SOO. All of these three oils passed the test to impart efficient activity against bacteria. Table 11 shows the antibacterial activity by Kirby Bauer method and here we found highest 32 $\mathrm{mm}$ and minimum $14 \mathrm{~mm}$ zone of inhibition. By the Figure 2 graph, it is clear that for all isolates of Escherichia coli, Pseudomonas aeruginosa, Pseudomonas luteola, Klebsiella pheumoniae, Staphylococcus aureus, Bacillus subtilis, TTO showed the highest antibacterial activity. It worked the most against Pseudomona luteola and Escherichia coli. SSO

\begin{tabular}{|c|c|c|c|c|c|c|c|}
\hline Test & 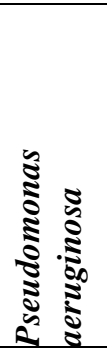 & 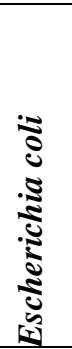 & 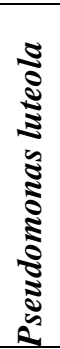 & 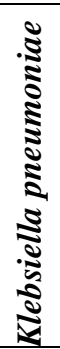 & Test & 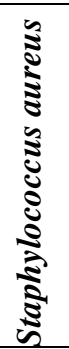 & 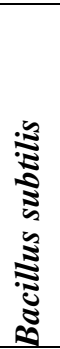 \\
\hline \multicolumn{5}{|c|}{ Gram negative bacteria } & \multicolumn{3}{|c|}{ Gram positive bacteria } \\
\hline APPA & - & - & - & - & APPA & - & - \\
\hline $\mathrm{H}_{2} \mathrm{~S}$ & - & - & - & - & $\mathrm{H}_{2} \mathrm{~S}$ & - & - \\
\hline BGLU & $(-)$ & - & - & + & BGLU & - & - \\
\hline ProA & + & - & + & + & ProA & + & \\
\hline SAC & - & - & - & + & SAC & + & + \\
\hline ILATk & + & + & + & + & ILATk & + & + \\
\hline GlyA & + & - & - & + & NAG & + & \\
\hline O129R & + & ND & + & + & O129R & + & \\
\hline ADO & - & - & - & + & NOVO & - & ND \\
\hline
\end{tabular}
and Ylang-ylang oil showed varying results with minimum activity for Staphylococcus aureus by Ylang-ylang oil.

Table 1. Biochemical identification of bacteria collected from different sources. 
International Advanced Research Journal in Science, Engineering and Technology

Vol. 6, Issue 6, June 2019

\begin{tabular}{|c|c|c|c|c|}
\hline BNAG & - & - & - & - \\
\hline dMAL & - & + & - & + \\
\hline LIP & - & - & - & - \\
\hline dTAG & - & - & - & - \\
\hline AGLU & - & - & - & - \\
\hline ODC & - & + & - & - \\
\hline GGAA & + & $\mathrm{ND}$ & - & - \\
\hline PyrA & + & - & - & + \\
\hline AGLTp & - & - & - & $(+)$ \\
\hline dMAN & - & + & - & + \\
\hline PLE & - & - & - & + \\
\hline dTRE & - & + & - & + \\
\hline SUCT & + & + & + & + \\
\hline LDC & - & + & - & + \\
\hline IMLTa & + & $\mathrm{ND}$ & + & + \\
\hline IARL & - & - & - & - \\
\hline dGLU & + & + & + & + \\
\hline dMNE & + & + & + & + \\
\hline TyrA & + & - & + & + \\
\hline CIT & + & - & + & + \\
\hline NAGA & - & - & - & + \\
\hline IHISa & - & - & + & + \\
\hline ELLM & - & $\mathrm{ND}$ & - & - \\
\hline dCEL & - & - & - & + \\
\hline GGT & + & - & + & + \\
\hline BXYL & - & - & - & + \\
\hline URE & - & - & - & + \\
\hline MNT & + & - & + & + \\
\hline AGAL & - & + & - & + \\
\hline CMT & + & + & + & - \\
\hline ILATa & + & ND & + & + \\
\hline BGAL & - & + & - & + \\
\hline OFF & - & + & - & + \\
\hline BAlap & + & - & & \\
\hline dSOR & - & + & - & \\
\hline $5 \mathrm{KG}$ & - & + & - & + \\
\hline PHOS & - & - & - & - \\
\hline BGUR & $(-)$ & - & - & - \\
\hline
\end{tabular}

\begin{tabular}{|l|l|l|}
\hline LAC & - & - \\
\hline dMAL & + & \\
\hline BGURr & - & \\
\hline AGLU & - & - \\
\hline dGAL & + & ND \\
\hline dRIB & - & + \\
\hline PyrA & + & - \\
\hline dRAF & - & - \\
\hline dMAN & + & - \\
\hline dXYL & ND & - \\
\hline dTRE & + & + \\
\hline dMNE & + & - \\
\hline TyrA & - & \\
\hline URE & - & + \\
\hline AGAL & - & - \\
\hline BGAL & - & + \\
\hline dSOR & - & - \\
\hline PHOS & + & + \\
\hline BGUR & - & + \\
\hline
\end{tabular}

1. $\quad$ ADONITOL $=\mathrm{ADO}$,

L-Pyrrolydonyl-ARYLAMIDASE=PyrA,

L-ARABITOL=IARL,

DCELLOBIOSE $=\mathrm{dCEL}, \quad$ BETA-GALACTOSIDASE $=\mathrm{BGAL}, \quad \mathrm{H}_{2} \mathrm{~S}$ production $=\mathrm{H}_{2} \mathrm{~S}, \quad$ BETA-N-ACETYLGLUCOSAMINIDASE=BNAG, Glutamyl Arylamidase pNA=AGLTp, D-GLUCOSE=dGLU, GAMMA-GlutamylTRANSFERASE=GGT, FERMENTATION/GLUCOSE=OFF, BETA-GLUCOSIDASE=BGLU, DMALTOSE $=\mathrm{dMAL}$, D-MANNITOL=dMAN, D-MANNOSE_dMNE, BETA-XYLOSIDASE=BXYL, BETA-Alanine arylamidase pNA=BALap, L-Proline ARYLAMIDASE=ProA, LIPASE=LIP, PALATINOSE=PLE, Tyrosine ARYLAMIDASE=TyrA, UREASE=URE, D-SORBITOL=dSOR, D-TAGATOSE=dTAG, D-TREHALOSE=dTRE, CITRATE(SODIUM)=CIT, MALONATE=MNT, 5-KETO-D-GLUCONATE $=5 \mathrm{KG}, \quad$ L-LACTATE alkanization=ILATk, ALPHA-GLUCOSIDASE=AGLU, SUCCINATE alkanization=SUCT, Beta-N-ACETYLGALACTOSEAMINIDASE=NAGA， ALPHA-GALACTOSIDASE=AGAL， PHOSPHATASE=PHOS， Glycine 


\section{International Advanced Research Journal in Science, Engineering and Technology}

Vol. 6, Issue 6, June 2019

ARYLAMIDASE=GlyA， ORNITHINE DECARBOXYLASE=ODC, LYSINE DECARBOXYLASE=LDC, L HISTIDINE assimilation=lHISa, COUMARATE=CMT, BETA-GLUCORONIDASE=BGUR, O/129 RESISTANCE (comp. vibrio.)=O129R, Glu-Gly-Arg-ARYLAMIDASE=GGAA, L-MALATE assimilation=1MLTa, L-LACTATE assimilation=lLATa, D_XYLOSE=dXYL， BETA GLUCORONIDASE=BGURr， D-GALACTOSE=dGAL, LACTOSE=LAC, N-ACETYL-D-GLUCOSAMINE=NAG, NOVOBIOCIN RESISTANCE=NOVO, DRAFFINOSE $=d R A F, D-T R E H A L O S E=d T R E, D-R I B O S E=d R I B$.

Table 2. Antibiotic susceptibility test of the bacterial isolates (according to CLSI guideline).

\begin{tabular}{|c|c|c|c|c|c|c|c|}
\hline Antibiotics & 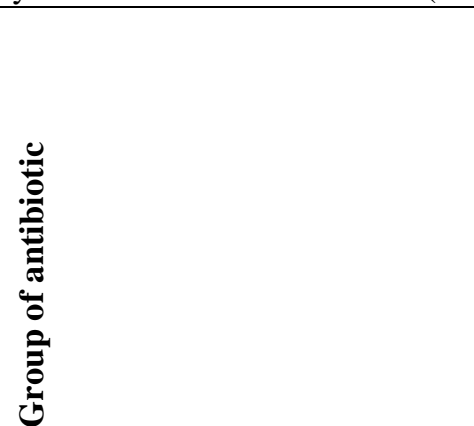 & 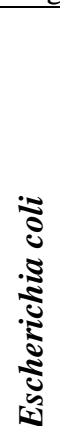 & 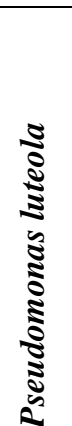 & 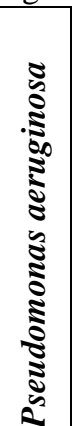 & 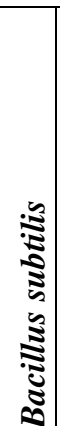 & 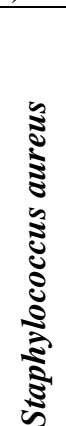 & 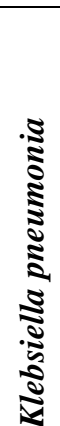 \\
\hline Nitrofurantoin $(100 \mu \mathrm{g})$ & Macrobid & $\mathrm{S}$ & - & - & - & - & $\mathrm{S}$ \\
\hline Cefepime $(30 \mu \mathrm{g})$ & Cephalosporins $\left(4^{\text {th }}\right)$ & $\mathrm{R}$ & $\mathrm{S}$ & $\mathrm{S}$ & - & - & $\mathrm{S}$ \\
\hline Gentamycin $(10 \mu \mathrm{g})$ & Aminoglycosides & $\mathrm{S}$ & S & $\mathrm{S}$ & S & S & $\mathrm{S}$ \\
\hline Piperacillin/Tazobactam $(100 / 10 \mu \mathrm{g})$ & Piperacillin//lactamase inhibitor & $\mathrm{S}$ & $S$ & $\mathrm{~S}$ & - & - & $\mathrm{S}$ \\
\hline Cefuroxime $(30 \mu \mathrm{g})$ & Cephalosporins $\left(2^{\text {nd }}\right)$ & $\mathrm{R}$ & - & - & - & - & $\mathrm{S}$ \\
\hline Cephradine $(30 \mu \mathrm{g})$ & Cephalosporins & - & - & - & $\mathrm{S}$ & $\mathrm{S}$ & - \\
\hline Colistin $(10 \mu \mathrm{g})$ & Polymixins & $\mathrm{S}$ & $\mathrm{R}$ & $\mathrm{S}$ & - & - & $\mathrm{S}$ \\
\hline Amoxicillin $(30 \mu \mathrm{g})$ & Aminobenzyl penicillin & $\mathrm{S}$ & - & - & - & - & $\mathrm{S}$ \\
\hline Amikacin $(30 \mu \mathrm{g})$ & Aminoglycosides & $\mathrm{S}$ & $\mathrm{S}$ & $\mathrm{S}$ & - & $\mathrm{S}$ & $\mathrm{S}$ \\
\hline Ampicillin $(10 \mu \mathrm{g})$ & Aminobenzyl penicillin & - & - & - & $\mathrm{S}$ & $\mathrm{S}$ & $\mathrm{R}$ \\
\hline Meropenem $(10 \mu \mathrm{g})$ & Carbapenems & $\mathrm{S}$ & $\mathrm{S}$ & $\mathrm{S}$ & - & - & $\mathrm{S}$ \\
\hline Ertapenem $(10 \mu \mathrm{g})$ & Carbapenems & $\mathrm{S}$ & - & - & - & - & $\mathrm{S}$ \\
\hline Cefoperazone/Sulbactam $(75 / 30 \mu \mathrm{g})$ & Blactamase inhibitor & $\mathrm{S}$ & $\mathrm{S}$ & $\mathrm{S}$ & - & - & $\mathrm{S}$ \\
\hline Trimethoprim/Sulfamethoxazole & Trimethoprim/ Sulfonamide & $\mathrm{S}$ & $S$ & - & - & - & $\mathrm{S}$ \\
\hline Ciprofloxacin $(5 \mu \mathrm{g})$ & Quinolones $\left(2^{\text {nd }}\right)$ & $\mathrm{R}$ & $\mathrm{S}$ & $\mathrm{S}$ & - & - & $\mathrm{S}$ \\
\hline Imipenem $(10 \mu \mathrm{g})$ & Carbapenems & $\mathrm{S}$ & $\mathrm{S}$ & $\mathrm{S}$ & - & - & $\mathrm{S}$ \\
\hline Neomycin $(30 \mu \mathrm{g})$ & Amynoglycoside & - & - & - & $\mathrm{S}$ & $\mathrm{S}$ & - \\
\hline Tetracycline $(30 \mu \mathrm{g})$ & Tetracyclines & - & & - & $\mathrm{S}$ & $\mathrm{S}$ & - \\
\hline Rifampicin $(5 \mu \mathrm{g})$ & Ansamycins & - & - & - & $\mathrm{S}$ & $\mathrm{S}$ & - \\
\hline Ceftriaxone $(30 \mu \mathrm{g})$ & Cephalosporins $\left(3^{\text {rd }} \& 4^{\text {th }}\right)$ & $\mathrm{R}$ & $\mathrm{S}$ & - & - & - & $\mathrm{S}$ \\
\hline Erythromycin $(15 \mu \mathrm{g})$ & Macrolides & - & - & - & $\mathrm{S}$ & $\mathrm{S}$ & - \\
\hline Cefpodoxime $(30 \mu \mathrm{g})$ & Cephalosporins $\left(3^{\text {rd }} \& 4^{\text {th }}\right)$ & - & - & - & $\mathrm{S}$ & $\mathrm{S}$ & - \\
\hline Tigecycline $(15 \mu \mathrm{g})$ & Glycylcyclines & $\mathrm{S}$ & $\mathrm{S}$ & $\mathrm{R}$ & - & - & $\mathrm{S}$ \\
\hline Nalidixic Acid $(30 \mu \mathrm{g})$ & Fluoroquinolones $\left(1^{\text {st }}\right)$ & $\mathrm{R}$ & - & - & - & - & $\mathrm{S}$ \\
\hline Novobiocin $(30 \mu \mathrm{g})$ & Aminocoumarin & - & - & - & $\mathrm{S}$ & - & - \\
\hline
\end{tabular}

Table 3. Total viable bacterial count of Pseudomonas aeruginosa in BHIB and BHIB with essential oils

\begin{tabular}{|c|c|c|c|c|c|c|c|c|}
\hline Dilution & 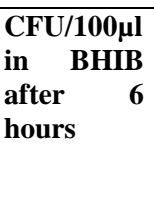 & 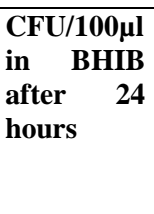 & 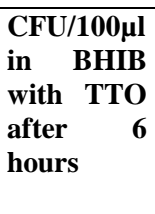 & 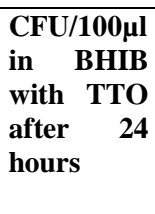 & $\begin{array}{lr}\text { CFU/100 } \mu l \\
\text { in } \quad \text { BHIB } \\
\text { with SOO } \\
\text { after } \\
\text { hours }\end{array}$ & 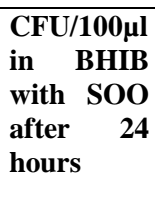 & 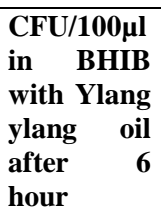 & $\begin{array}{l}\text { CFU/100 } \mu \mathrm{l} \\
\text { in BHIB } \\
\text { with Ylang } \\
\text { ylang oil } \\
\text { after } 24 \\
\text { hour }\end{array}$ \\
\hline $10^{-1}$ & TNTC & TNTC & 3 & 65 & TNTC & TNTC & 41 & 145 \\
\hline $10^{-2}$ & TNTC & TNTC & 0 & 48 & TNTC & TNTC & 15 & 100 \\
\hline $10^{-3}$ & 220 & TNTC & 0 & 26 & 200 & 280 & 8 & 40 \\
\hline $10^{-4}$ & 70 & TNTC & 0 & 0 & 35 & 185 & 0 & 11 \\
\hline $10^{-5}$ & 13 & 290 & 0 & 0 & 12 & 33 & 0 & 0 \\
\hline
\end{tabular}


Vol. 6, Issue 6, June 2019

Table 4. Total viable bacterial count of Pseudomonas luteola in BHIB and BHIB with essential oils

\begin{tabular}{|c|c|c|c|c|c|c|c|c|}
\hline Dilution & 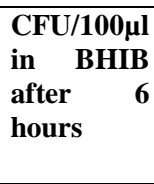 & $\begin{array}{l}\text { CFU/100 } \mu \text { l } \\
\text { in BHIB } \\
\text { after } 24 \\
\text { hours }\end{array}$ & 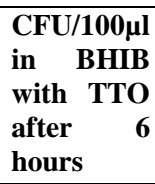 & 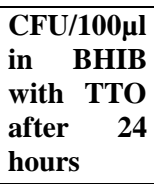 & 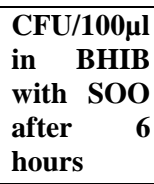 & 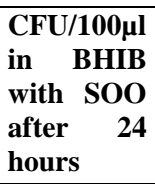 & 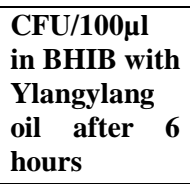 & 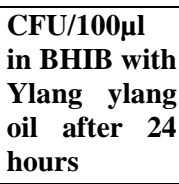 \\
\hline $10^{-1}$ & 120 & TNTC & 28 & 24 & 10 & 60 & 87 & TNTC \\
\hline $10^{-2}$ & 48 & TNTC & 14 & 18 & 3 & 35 & 36 & 230 \\
\hline $10^{-3}$ & 9 & TNTC & 9 & 8 & 0 & 7 & 8 & 150 \\
\hline $10^{-4}$ & 0 & TNTC & 0 & 0 & 0 & 0 & 0 & 78 \\
\hline $10^{-5}$ & 0 & 236 & 0 & 0 & 0 & 0 & 0 & 17 \\
\hline
\end{tabular}

Table 5. Total viable bacterial count of Staphylococcus aureus in BHIB and BHIB with essential oils

\begin{tabular}{|c|c|c|c|c|c|c|c|c|}
\hline Dilution & 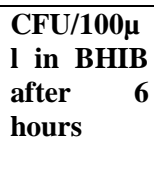 & $\begin{array}{l}\text { CFU/100 } \mu \\
1 \text { in BHIB } \\
\text { after } 24 \\
\text { hours }\end{array}$ & 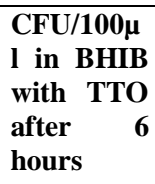 & 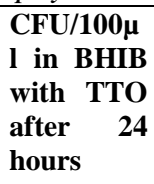 & 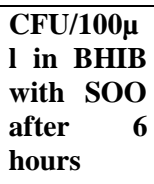 & 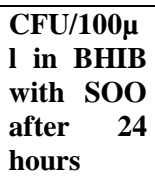 & 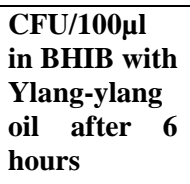 & 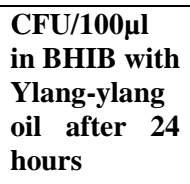 \\
\hline $10^{-1}$ & 132 & TNTC & 46 & 18 & 118 & TNTC & 122 & TNTC \\
\hline $10^{-2}$ & 94 & TNTC & 20 & 10 & 89 & 220 & 81 & TNTC \\
\hline $10^{-3}$ & 66 & TNTC & 3 & 0 & 56 & 190 & 40 & 160 \\
\hline $10^{-4}$ & 42 & 280 & 0 & 0 & 12 & 75 & 8 & 42 \\
\hline $10^{-5}$ & 18 & 140 & 0 & 0 & 0 & 14 & 0 & 16 \\
\hline
\end{tabular}

Table 6. Total viable bacterial count of Escherichia coli in BHIB and BHIB with essential oils

\begin{tabular}{|c|c|c|c|c|c|c|c|c|}
\hline Dilution & 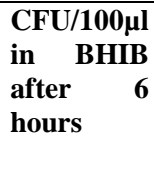 & $\begin{array}{l}\text { CFU/100 } \mu \text { l } \\
\text { in BHIB } \\
\text { after } 24 \\
\text { hours }\end{array}$ & 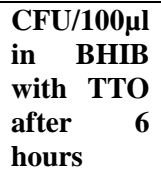 & 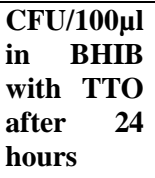 & 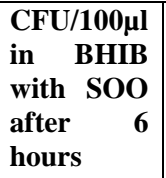 & 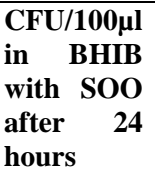 & 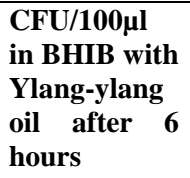 & 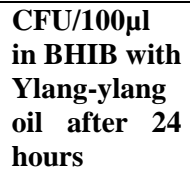 \\
\hline $10^{-1}$ & TNTC & TNTC & 44 & 70 & 110 & TNTC & 38 & 236 \\
\hline $10^{-2}$ & TNTC & TNTC & 35 & 45 & 64 & 216 & 25 & 168 \\
\hline $10^{-3}$ & TNTC & TNTC & 11 & 32 & 13 & 180 & 16 & 78 \\
\hline $10^{-4}$ & 270 & TNTC & 0 & 13 & 0 & 52 & 0 & 56 \\
\hline $10^{-5}$ & 150 & 290 & 0 & 0 & 0 & 6 & 0 & 25 \\
\hline
\end{tabular}

Table 7. Total viable bacterial count of Klebsiella pneumoniae in BHIB and BHIB with essential oils

\begin{tabular}{|c|c|c|c|c|c|c|c|c|}
\hline Dilution & 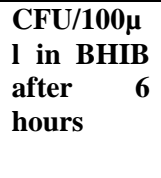 & 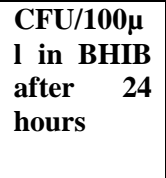 & 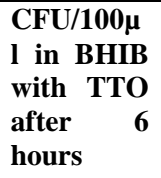 & 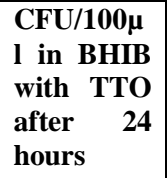 & 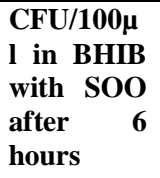 & 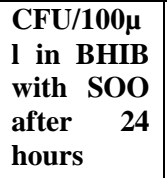 & \begin{tabular}{lr}
\multicolumn{2}{c}{ CFU/100 $\mu \mathrm{l}$} \\
in $\quad$ BHIB \\
with Ylang- \\
ylang r oil \\
after 6 hours
\end{tabular} & $\begin{array}{l}\text { CFU/100ul in } \\
\text { BHIB with } \\
\text { Ylang-ylang } \\
\text { oil after } 24 \\
\text { hours }\end{array}$ \\
\hline $10^{-1}$ & TNTC & TNTC & 76 & 110 & TNTC & TNTC & 72 & TNTC \\
\hline $10^{-2}$ & TNTC & TNTC & 55 & 90 & 88 & TNTC & 25 & TNTC \\
\hline $10^{-3}$ & TNTC & TNTC & 28 & 56 & 30 & 290 & 15 & 70 \\
\hline $10^{-4}$ & TNTC & TNTC & 2 & 14 & 4 & 126 & 0 & 48 \\
\hline $10^{-5}$ & 90 & 286 & 0 & 0 & 0 & 15 & 0 & 12 \\
\hline
\end{tabular}

Table 8. Total viable bacterial count of Bacillus subtilis in BHIB and BHIB with essential oils

\begin{tabular}{|c|c|c|c|c|c|c|c|c|}
\hline Dilution & 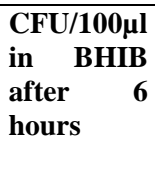 & 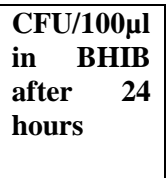 & 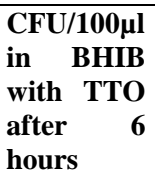 & 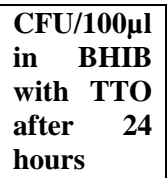 & 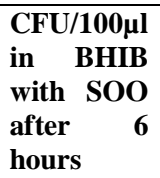 & 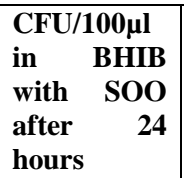 & $\begin{array}{l}\text { CFU/100 } \mu \mathrm{l} \\
\text { in BHIB with } \\
\text { Ylang-ylang } \\
\text { oil after } 6 \\
\text { hours }\end{array}$ & 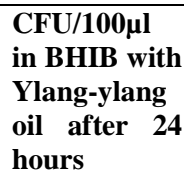 \\
\hline $10^{-1}$ & 78 & 217 & 46 & 83 & 70 & 204 & 65 & 188 \\
\hline $10^{-2}$ & 60 & 124 & 32 & 58 & 54 & 112 & 56 & 112 \\
\hline $10^{-3}$ & 41 & 78 & 18 & 41 & 32 & 66 & 33 & 68 \\
\hline $10^{-4}$ & 32 & 56 & 6 & 28 & 21 & 41 & 20 & 41 \\
\hline $10^{-5}$ & 17 & 42 & 0 & 0 & 11 & 34 & 7 & 31 \\
\hline
\end{tabular}




\section{International Advanced Research Journal in Science, Engineering and Technology}

Vol. 6, Issue 6, June 2019

Calculation of inhibition percentage:

$$
\begin{aligned}
& \text { CFU }=\frac{\text { Number of colonies } \times \text { reciprocal of the dilution factor }}{\text { Volume of plated suspension }} \\
& \text { Inhibition percentage }=1-\left(\frac{\text { CFU in oil }}{\text { CFU in broth }}\right) \times 100
\end{aligned}
$$

Table 9. Inhibition percentage of TTO, SOO and Ylang-ylang oil

\begin{tabular}{|l|l|l|l|l|l|l|}
\hline \multirow{2}{*}{ Bacteria } & \multicolumn{2}{|c|}{ TTO } & \multicolumn{2}{c|}{ SOO } & \multicolumn{2}{c|}{ Ylang-ylang Oil } \\
\cline { 2 - 7 } & $\begin{array}{l}\text { After 6 hours } \\
\text { incubation }\end{array}$ & $\begin{array}{l}\text { After 24 hours } \\
\text { incubation }\end{array}$ & $\begin{array}{l}\text { After 6 hours } \\
\text { incubation }\end{array}$ & $\begin{array}{l}\text { After 24 hours } \\
\text { incubation }\end{array}$ & $\begin{array}{l}\text { After 6 hours } \\
\text { incubation }\end{array}$ & $\begin{array}{l}\text { After 24 hours } \\
\text { incubation }\end{array}$ \\
\hline $\begin{array}{l}\text { Pseudomonas } \\
\text { aeruginosa }\end{array}$ & 100 & 100 & 50 & 89 & 97 & 100 \\
\hline $\begin{array}{l}\text { Pseudomonas } \\
\text { luteola }\end{array}$ & 77 & 100 & 80 & 100 & 27 & 93 \\
\hline Escherichia coli & 100 & 100 & 81 & 100 & 80 & 100 \\
\hline $\begin{array}{l}\text { Staphylococcus } \\
\text { aureus }\end{array}$ & 66 & 100 & 16 & 74 & 81 & 89 \\
\hline Bacillus subtilis & 42 & 62 & 6 & 11 & 17 & 27 \\
\hline $\begin{array}{l}\text { Klebsiella } \\
\text { pneumonia }\end{array}$ & 100 & 100 & 95 & 100 & 96 & 100 \\
\hline
\end{tabular}

Table 10. Average inhibition percentage of TTO, SOO and Ylang-ylang oil

\begin{tabular}{|l|l|l|}
\hline \multirow{2}{*}{ Essential oils } & \multicolumn{2}{c|}{ Average percentage inhibition } \\
\cline { 2 - 3 } & After 6 hours incubation & After 24 hours incubation \\
\hline Tea Tree Oil (TTO) & $80.83 \%$ & $93.67 \%$ \\
\hline Sweet Orange Oil (SOO) & $59.5 \%$ & $74.16 \%$ \\
\hline Ylang-ylang Oil & $70.33 \%$ & $81.33 \%$ \\
\hline
\end{tabular}

Table 11. Comparing the effectiveness of essential oils by agar well diffusion method

\begin{tabular}{|l|l|l|l|}
\hline Bacteria & TTO & SOO & Ylang-ylang Oil \\
\hline Pseudomonas aeruginosa & $26 \mathrm{~mm}$ & $20 \mathrm{~mm}$ & $18 \mathrm{~mm}$ \\
\hline Pseudomonas luteola & $32 \mathrm{~mm}$ & $20 \mathrm{~mm}$ & $20 \mathrm{~mm}$ \\
\hline Escherichia coli & $30 \mathrm{~mm}$ & $16 \mathrm{~mm}$ & $24 \mathrm{~mm}$ \\
\hline Staphylococcus aureus & $20 \mathrm{~mm}$ & $18 \mathrm{~mm}$ & $10 \mathrm{~mm}$ \\
\hline Klebsiella pneumoniae & $22 \mathrm{~mm}$ & $18 \mathrm{~mm}$ & $14 \mathrm{~mm}$ \\
\hline Bacillus subtilis & $25 \mathrm{~mm}$ & $20 \mathrm{~mm}$ & $20 \mathrm{~mm}$ \\
\hline
\end{tabular}

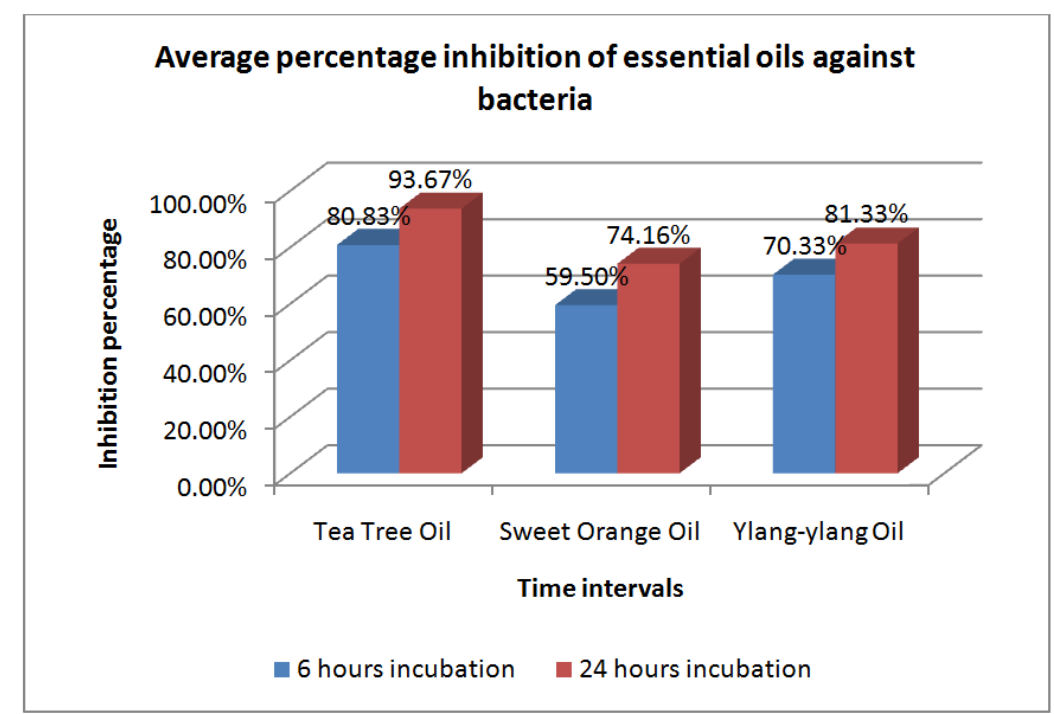

Figure 1. Comparison of average percentage inhibition of essential oils. 


\title{
International Advanced Research Journal in Science, Engineering and Technology
}

\author{
Vol. 6, Issue 6, June 2019
}

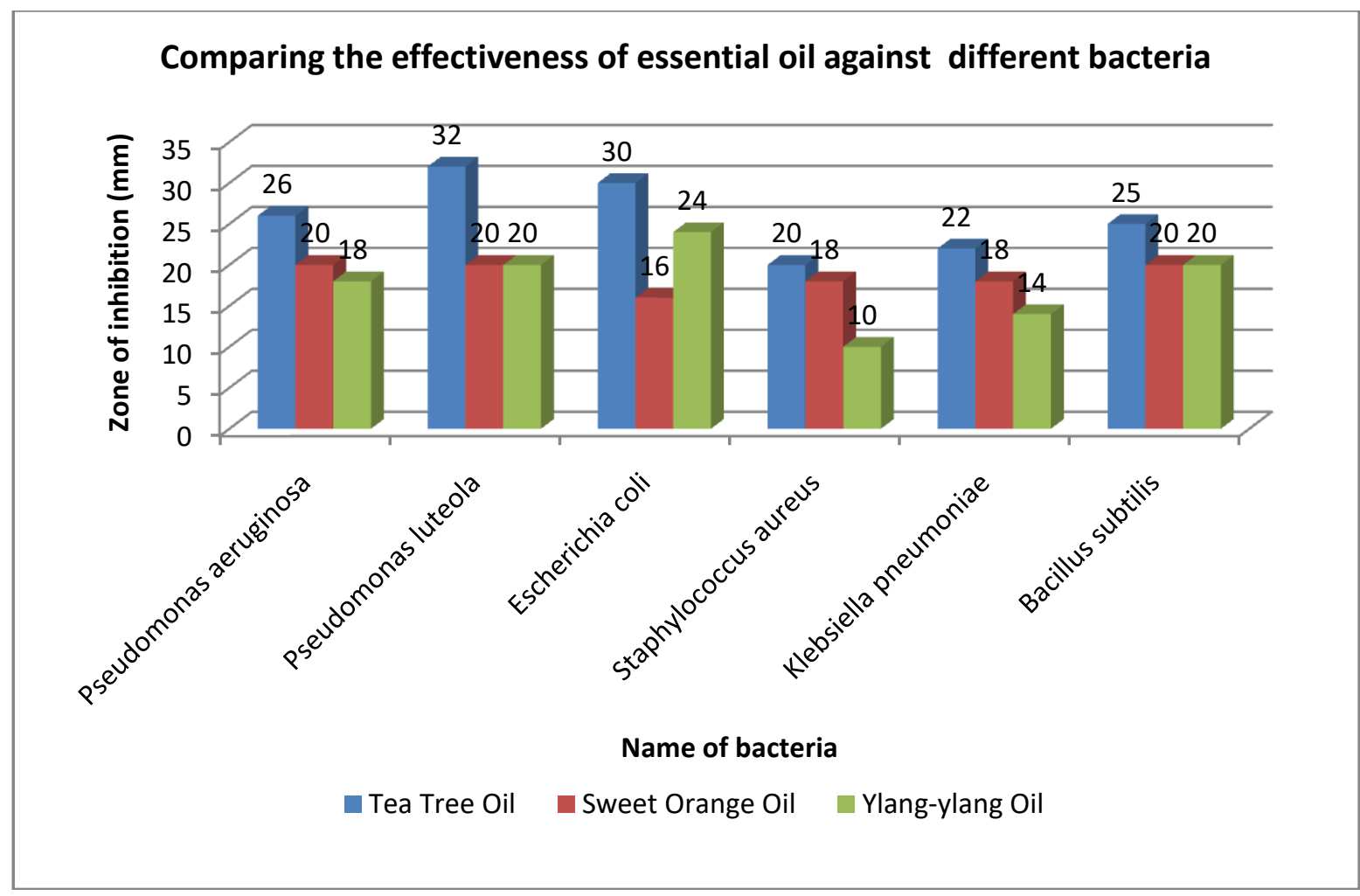

Fig2. Comparison among the effectiveness of essential oils against six different bacteria by agar well diffusion method.

\section{DISCUSSION}

To investigate the antibacterial activity of three essential oils from tea tree, sweet orange and ylang-ylang, some clinical, environmental and laboratory freeze dried isolates were selected. Do to the abuse of antibiotics, from the last few decades many bacteria stopped responding with antibiotic treatments and new approaches are under investigation to find alternatives to combat diseases caused by such antibiotic resistant bacteria. Plants and their extract are used from the prehistoric era to treat disease generally, but little was known to use a specific extract for treating specific disease. Essential oils possess antimicrobial properties and in the current context we compared the activities of TTO, SOO and Ylang-ylang oil against six different bacterial isolates specifically.

To start our research at first we needed to choose some bacteria and identify them properly as well as their antibiotic susceptibility pattern to find whether the essential oils works with the antibiotic resistant isolates as we concern about the resistant bacteria which can be inhibited by essential oils. Here after biochemical identification we confirmed the identification of six bacteria like Escherichia coli, Pseudomonas aeruginosa, Pseudomonas luteola, Klebsiella pheumoniae, Staphylococcus aureus, Bacillus subtilis.

During the study of antibiotic susceptibility of the selected bacteria we found Escherichia coli was resistant to $1^{\text {st }}$ generation Fluoroquinolones and to a few $2^{\text {nd }}, 3^{\text {rd }}$ and even $4^{\text {th }}$ generation cephalosporin drugs. Other bacterial isolates showed sensitivity to some antibiotics as well as resistance to some antibiotics. It can easily be understood that the infection caused by such multi drug resistant Escherichia coli is beyond the capability of treatment using antibiotics. Environmental and laboratory freeze dried isolates showed sensitivity toward the antibiotics we used during this study for them. The reason for such sensitivity might be they never have encountered with antibiotics before, they have not exposed the resistance genes from other drug resistant isolates.

After studying the antibiotic sensitivity test, we further attempted to determine the antibacterial activity of TTO, SOO and ylang-ylang oil both by agar well diffusion and broth dilution method. From the results we can see the similarities found in both methods applied for detection of antibacterial activity. From table 3 to table 8 we found the reduction of CFU after incubation with essential oils than the CFU from samples without essential oil. We found such results for all of the six bacterial isolates and all the three essential oils. The inhibition of growth was further calculated as percentage inhibition and there found the percentage of the inhibiting capacity of the oils against these bacteria (Table 9). Most effective essential oil was TTO which imparted inhibiting activity after 6 hours for Escherichia coli, Pseudomonas 


\title{
International Advanced Research Journal in Science, Engineering and Technology
}

\author{
Vol. 6, Issue 6, June 2019
}

aeruginosa, Pseudomonas luteola, Klebsiella pheumoniae, Staphylococcus aureus, Bacillus subtilis was ranging from $100 \%$ to $42 \%$ and after 24 was it ranged from $100 \%$ to $62 \%$. SOO showed inhibiting capacity after 6 hours $100 \%$ to $11 \%$ and after 24 hours from 100\% to $6 \%$ (the lowest activity was for Bacillus subtilis). Finally Ylang-ylang oil had the inhibition percentage of $100 \%$ to $10 \%$ at 6 hours and $100 \%$ to $27 \%$ at 24 hours incubation. All the oils exhibited quiet remarkable results showing inhibiting capabilities except Bacillus subtilis. It showed the lowest degree of sensitivity towards all the oils. As it is still sensitive towards the conventional antibiotics, our concern was with the drug resistant isolates. Pseudomonas aeruginosa, Klebsiella pneumoniae and Escherichia coli having higher degrees of antibiotic resistance showed satisfactory result with TTO, SOO and Ylang-ylang oil. These oils can be used to treat infections caused by these bacteria specifically.

\section{CONCLUSION}

At present medical science is facing problem to treat infectious diseases with antibiotics due to the multi drug resistance properties of the bacteria. As a result, these bacteria can now cause life threatening conditions being nonresponsive toward the antibiotics. So searching for alternatives especially from plant origin (due to less toxicity, availability) is going on to treat people infected with such antibiotic resistant bacteria. In our current study we observed that TTO has the highest ability to inhibit all of the six selected bacteria which are already resistant to antibiotics. The most significant result was found for Escherichia coli (having resistance to $4^{\text {th }}$ generation antibiotics) towards all of these three essential oils. This breakthrough information can be aimed to develop new drugs for treating $4^{\text {th }}$ generation cephalosporin resistant bacteria.

All paragraphs must be indented. All paragraphs must be justified, i.e. both left-justified and right-justified.

\section{ACKNOWLEDGMENT}

Authors are thankful to the Microbiology Laboratory, Department of Microbiology, Stamford University Bangladesh for technical support during the study.

\section{REFERENCES}

[1]. Shah A, Jani M, Shah H, Chaudhary N and Shah A. 2014. Antimicrobial effect of Clove oil (Laung) extract on Enterococcus faecalis. Journal of Advanced Oral Research. 5(3): 1-3.

[2]. Raut R R, Sawant A R, Jamge B B. 2014. Antimicrobial activity of Azadirachta indica (Neem) against pathogenic microorganisms, Journal of Academia and Industrial Research. 3(7): 327-329.

[3]. Bassolé, I. H. N. and Juliani, H. R., (2012). Essential oils in combination and their antimicrobial properties. Mol., 17: $3989-4006$.

[4]. Rana I S, Rana A S and Rajak R C. 2011. Evaluation of Antifungal Activity in Essential Oil of the Syzygium aromaticum (L.) by Extraction, Purification and Analysis of its Main Component Eugenol, Brazer Journal of Microbiology. 42(4): 1269-77.

[5]. Srivastava S, Cahill D M, Conlan XA and Adholeya A. 2014. A Novel In Vitro Whole Plant System for Analysis of Polyphenolics and their Antioxidant Potential in Cultivars of Ocimum basilicum, Journal of Agricultural and Food Chemistry. 62(41): $10064-10075$.

[6]. Sharma A, Sharma V, Kumawat TK, Seth R. 2014. A Review on Antidermatophytic Efficiency of Plant Essential Oils.International Journal of Pure and Applied Bioscience.2(6):265-278.

[7]. Cosentinos Barra A, Pisano B, Cabizzam Pirrisi FM and Palmas F. 2003. Composition and Antimicrobial Properties of Sardinian Juniperus Essential Oil Against Food Borne Pathogens and Spoilage Microorganisms, Journal of Food Science and Technology. 66 : 1288 - 1291.

[8]. Burt SA. 2004. Essential Oils: Their Antibacterial Properties and Potential Applications in Food - A Review, International Journal of Food Microbiology. 94: 223-253.

[9]. Bajpai VK, Baek HK and Kang SC. 2012. Control of Salmonella in foods by using essential oils: A review. Food Res. Int., $45: 722-734$.

[10]. Cavaleiro C, Salgueiro L, Goncalves M J, Hrimplpeng K, Pinto J and Pinto E. 2015. Antifungal activity of the essential oil of Angelica major against Candida, Cryptococcus, Aspergillus and dermatophyte species, Journal of Natural Medicines. 69:241-248.

[11]. Venturi C R, Danielli L J, Klein F, Apel M A, Montanha J A, Bordignon SL, Roehe P M, Fuentefria A M and Henriques A T. 2015. Chemical analysis and in vitro antiviral and antifungal activities of essential oils from Glechon spathulata and Glechon marifolia, Pharmaceutical Biology. 53(5): 682-688.

[12]. Thusoo S, Gupta S, Sudan R, Kour J, Bhagat S, Hussain R and Bhagat M. 2014. Antioxidant activity of essential oil and extracts of Valeriana jatamansi roots, Biology and Medicinal Research International. 1-4.

[13]. Baptista EB, Zimmermann-Franco DC, Lataliza AAB, Raposo NRB. 2015. Chemical composition and antifungal activity of essential oil from Eucalyptus smithii against dermatophytes. Revista da Sociedade Brasileira de Medicina Tropical. 48(6).

[14]. Marzougui N, Boubaya A, Thabti I, Ferchichi A and Bakhrouf A. 2016. Antibacterial Activity of Extracts of Diploid and Induced Autotetraploid Tunisian Populations of Trigonella foenum-graecum L. Journal of Medicinal Plants Research. 6(38): 5166-72.

[15]. Menchaca Md C V, Morales C R, Star J V, Cárdenas A, Morales M R and González M A N. 2016. Antimicrobial Activity of Five Plants from Northern Mexico on Medically Important Bacteria. Advanced Research Journal of Microbiology. 1: 060-066.

[16]. Nunkoo D H and Mahomoodally M F. 2016. Ethnopharmacological Survey of Native Remedies Commonly used Against Infectious Diseases in the Tropical Island of Mauritius, Journal of ethnopharmacology.143(2): 548-64.

[17]. Levy S B and Marshall B. 2007. Antibacterial Resistance Worldwide: Causes, Challenges and Responses, Nature Medicine Supplement. 10(12): 


\title{
International Advanced Research Journal in Science, Engineering and Technology
}

\author{
Vol. 6, Issue 6, June 2019
}

122-129.

[18]. Lin J, Nishino K, Roberts MC, Tolmasky M, Aminov RI \& Zhang L. 2015. Mechanisms of Antibiotic Resistance, Frontiers Microbiology.6:1-3

[19]. Hossain MA, Harbi S R A L, Weli Kumar H K, Chandana E, Preethi S and Chauhan J B. 2012. In vitro Antimicrobial Activity and Phytochemical Screening of Aloe Vera L., International Journal of Current Pharmaceutical. 4: 45- 47.

[20]. Gobalakrishnan R, Kulandaivelu M, Bhuvaneswari R, Kandavel D and Kannan L. 2013. Screening of Wild Plant Species for Antibacterial Activity and phytochemical analysis of Tragia involucrata L. J Pharm Anal. 3(6): 460-465.

[21]. Arellanes AJ, Mariana Meckes M, Raquel Ramirez R, Torres J. and Luna- Herrera J. 2003. Activity Against Multidrug-Resistant Mycobacterium tuberculosis in Mexican Plants Used to Treat Respiratory Diseases. Phytotherapy Research,17(8): 903-908.

[22]. Genersch E. 2009. American Foulbrood in Honeybees \& its Causative Agent, Paenibacillus larvae. Jour of Invertebrate Pathology.103(1): 10-19

[23]. Dorman H J D and Deans SG. 2000. Antimicrobial Agents from Plants, Antibacterial Activity of Plant Volatile Oils, Journal of Applied Microbiology. 88: 308-316.

[24]. Delaquis P J, Stanich K, Girarad B and Mazza G. 2002. Antimicrobial Activity of Individual and Mixed Fractions of Dill, Cilantro, Coriander and Eucalyptus Essential Oils, International Journal of Food Microbiology. 74(1-2): 101-109.

[25]. Benchaar C, Calsamiglia S, Chaves AV, Fraser GR, Colombatto D, McAllister TA and Beauchemin KA. 2008. A review of plant-derived essential oils in ruminant nutrition and production. Animal Feed Science Technology. 145: 209-228.

[26]. Freires I A, Denny C, Benso B, Alencar SM and Rosalen P. 2015. Antibacterial activity of essential oils and their isolated constituents against carcinogenic bacteria: A systematic review. Molecules. 20: 7329-7358.

[27]. Lezcano N, Nunez M, Espim I, Dupre A, Pinna P, Molicotti G, Fadda and S. Zanetti. 2000. Department of Science Biomedich Degli University Study, Sassari Italy.

[28]. Banes-Marshall L, Cawley P, Phillips CA. 2001. In vitro activity of Melaleuca alternifolia (tea tree) oil against bacterial and Candida spp. isolates from clinical specimens. Br J Biomed Sci 58: 139-145.

[29]. Hammer KA, Dry L, Johnson M, Michalak EM, Carson CF, et al. 2003. Susceptibility of oral bacteria to Melaleuca alternifolia (tea tree) oil in vitro. Oral Microbiol. Immunol 18: 389-392.

[30]. Andrade BFMT, Barbosa LN, Alves FCB, Albano M, Rall VLM, et al. 2016. The antibacterial effects of Melaleuca alternifolia, Pelargonium graveolens and Cymbopogon martinii essential oils and major compounds on liquid and vapor phase. J Essential Oil Res 28:227-233.

[31]. Hammer KA, Carson CF, Riley TV, Nielsen JB. 2006. A review of the toxicity of Melaleuca alternifolia (tea tree) oil. Food Chem Toxicol 44: 616-25.

[32]. Pazyar N, Yaghoobi R, Bagherani N, Kazerouni A. 2013. A review of applications of tea tree oil in dermatology. Inter J Dermatol. $52: 784-790$.

[33]. Carson CF, Mee BJ, Riley TV. 2002. Mechanism of action of Melaleuca alternifolia (tea tree) oil on Staphylococcus aureus determined by timekill, lysis, leakage, and salt tolerance assays and electron microscopy. Antimicrob Agents Chemother. 48: 1914-1920.

[34]. Siddique S, Shafique M, Parveen Z, Khan SJ, Khanum R. 2011. Volatile components, antioxidant and antimicrobial activity of Citrus aurantiumvar. bitter orange peel oil. Pharmacologyonline. 2: 499-507.

[35]. Adnan M, Umer A, Ahmad I, Hayat K, Shakeel SN. 2014. In vitro evaluation of biological activities of citrus leaf extracts. Sains Malaysiana. 43(2): 185-194.

[36]. Parashar S, Sharma H, Garg M. 2014. Antimicrobial and antioxidant activities of fruits and vegetable peels: A review. Journal of Pharmacognosy and Phytochemistry. 3(1): 160-164.

[37]. Kusuma I W, Murdiyanto Arung ET, Syafrizal Kim Y. 2014.Antimicrobial and antioxidant properties of medicinal plants used by the Bentian tribe from Indonesia. Food Science and Human Wellness. 3(3-4):191-196. doi: 10.1016/j.fshw.2014.12.004.

[38]. Nguyen-Pouplin J, Tran H, Tran H. et al. 2007. Antimalarial and cytotoxic activities of ethnopharmacologically selected medicinal plants from South Vietnam. Journal of Ethnopharmacology. 109(3):417-427. doi: 10.1016/j.jep.2006.08.011

[39]. Rahman MM, Lopa S S, Sadik G. et al. 2005. Antibacterial and cytotoxic compounds from the bark of Cananga odorata . Fitoterapia. 76(78):758-761. doi: 10.1016/j.fitote.2005.08.011. 